\title{
SITUAÇÃO ENDEMICA DA HANSENÍASE NO PERÍODO DE 2006 A 2011 NO MUNICÍPIO DE APARECIDA DE GOIÂNIA - GO
}

\author{
Maria Priscila Moraes dos Santos MACHADO ${ }^{1}$ \\ Rodrigo Cardoso DA SILVA ${ }^{2}$ \\ Alyne Leite Gomes NOGUEIRA ${ }^{3}$ \\ Cleiciane Vieira de Lima BARROS ${ }^{4}$
}

${ }^{1}$ Enfermeira e Licenciada em Biologia. Especialista em Enfermagem do Trabalho. Residente em infectologia no Hospital de Doenças Tropicais Dr. Anuar Auad - Goiânia - Goiás. E-mail: mprimachado@ hotmail.com.

${ }^{2}$ Enfermeiro. Docente na Escola de Técnico e Auxiliar de Enfermagem São Vicente de Paulo. Especializando em Enfermagem do Trabalho pelo Instituto Consciência Goiás (ICG). E-mail: enf.rodrigo1@ gmail.com

${ }^{3}$ Enfermeira do Hospital das Clínicas da Universidade Federal de Goiás (UFG). Mestre e Doutoranda em Enfermagem pela FEN-UFG. E-mail: alynenogueira@ hotmail.com.

${ }^{4}$ Enfermeira. Especialista em docência do ensino superior. Docente na Faculdade Alfredo Nasser (UNIFAN). Mestranda em Enfermagem pela FEN-UFG. E-mail: cleicianelima@gmail.com

\section{Recebido em: 27/05/2014 - Aprovado em: 15/09/2014 - Disponibilizado em: 15/12/2014}

RESUMO: A hanseníase é uma doença sistêmica, infectocontagiosa, crônica e curável. É um agravo de notificação compulsória e apresenta alta incidência e prevalência. É uma doença que por vezes negligenciada por clientes e profissionais de saúde. Diante disso, representa um importante problema de saúde pública. Este estudo teve por objetivo apresentar a situação da endemia da hanseníase no município de Aparecida de Goiânia-GO nos anos de 2006 a 2011. Trata-se de um estudo descritivo baseado em dados do Sistema de Informação de Agravos de Notificação (SINAN) do município. Considerando que os indicadores avaliados neste período apontam para uma manutenção da endemia hansênica no município estudado. Faz-se necessário o desenvolvimento de estudos científicos que contemple essa temática no estado de Goiás e especificamente na cidade de Aparecida de Goiânia. Contudo, é fundamental que haja intensificação das atividades de controle da doença, objetivando desenvolver estratégicas educativas com participação da comunidade, aprimoramento do sistema de informação e o compromisso efetivo dos gestores.

Palavras-chave: Hanseníase. Epidemiologia. Saúde Publica. Ciências da Saúde. Doenças Transmissíveis.

\begin{abstract}
Leprosy is a systemic, infectious, chronic and treatable disease . It is a bill of compulsory notification and shows high incidence and prevalence. It is a disease that often overlooked by clients and health professionals . Thus, it represents an important public health problem. This study aimed to present the situation of endemic leprosy in the city of Aparecida de Goiânia -GO in the years 2006-2011. This is a descriptive study based on data from the Information System for Notifiable Diseases ( SINAN ) the municipality. Whereas the indicators evaluated in this period show a maintenance of endemic leprosy in the municipality. It is necessary to develop scientific studies that addresses this issue in the state of Goiás and specifically in the city of Aparecida de Goiânia . However , it is crucial that intensification of disease control activities, aiming to develop educational strategies with community participation , improvement of the information system and effective commitment of managers.
\end{abstract}

Keywords: Leprosy. Epidemiology. Public Health. Health Sciences. Communicable Diseases. 


\section{INTRODUÇÃO}

A hanseníase é uma doença sistêmica, infectocontagiosa, crônica, curável e de grande importância para a saúde pública. Pode apresentar alterações imunológicas e também reumatológicas, causada pelo bacilo Mycobaterium Leprae, conhecido também como bacilo de Hansen. A transmissão ocorre pelo contato interpessoal e apresenta sinais e sintomas clínicos neurológicos específicos e característicos de cada uma das formas clínicas em que pode se apresentar esta enfermidade (BRASIL, 2008).

A hanseníase, a nível nacional e mundial, é uma doença que ainda apresenta elevadas taxas de prevalência e incidência, novos casos surgem todos os anos, entretanto o número desses casos é constante (FAÇANHA et al., 2006).

O controle desta patologia se configura em uma meta desafiante, visto que, $500 \mathrm{mil}$ novos casos surjam no mundo anualmente. $\mathrm{O}$ Brasil é o segundo país que apresenta as taxas mais altas de prevalência desta doença, sendo detectado em média, um novo caso a cada 12 minutos (CUNHA et al., 2012; JUNIOR, VIEIRA, CALDEIRA,2012).

Por ser uma doença de notificação compulsória em todo território nacional e de investigação obrigatória, os casos diagnosticados devem ser notificados, utilizando a ficha de notificação e investigação do Sistema de Informação
Nacional de Agravos (SINAN). (BRASIL, 2006; BRASIL, 2010; FREITAS, 2010).

Ao avaliar a prevalência da hanseníase nas regiões brasileiras, percebe-se que, a doença foi parcialmente erradicada nas regiões sul e sudeste. De acordo com Brasil (2011) estes resultados estão diretamente relacionados à alta cobertura do Programa de Agente Comunitário de Saúde (PACS) e as Estratégias de Saúde da Família (ESF), entretanto, as regiões norte, nordeste e centrooeste apresentam os índices mais elevados de hanseníase (BRASIL, 2012; JUNIOR, VIEIRA, CALDEIRA, 2012).

De acordo com dados do SINAN (2012), o coeficiente de prevalência de hanseníase referente ao ano de 2011 no Estado de Goiás foi de 2,35/10.000 habitantes, configurando-se como parâmetros médios de acordo com indicadores epidemiológicos. Já no município de Aparecida de Goiânia, o coeficiente de prevalência no ano de 2011 foi de 4,7/10.000 habitantes. Apesar de o coeficiente apresentar o dobro do Estado de Goiás ainda configurase como parâmetro médio, sendo assim, a prevalência de ambos configura-se como um sério problema de saúde pública.

De acordo com Ministério da Saúde (MS) coeficientes de prevalência entre 1,0 e 4,9/10.000 habitantes configura-se como parâmetros médio. Portanto, o levantamento destes dados adquire relevância na medida em 
que possibilita medir a magnitude da endemia, bem como a intensidade de detecção dos casos (BRASIL, 2010).

A hanseníase será considerada eliminada quando sua taxa de prevalência estiver menor que $1,0 / 10.000$ habitantes. No Brasil, para o alcance dessa taxa são implementadas estratégias, como a busca ativa de casos novos, vigilância de contatos, prevenção das incapacidades e reabilitação (FAÇANHA, 2006; BRASIL, 2011; JUNIOR, VIEIRA, CALDEIRA, 2012).

Diante do exposto, vale ressaltar a relevância da vigilância epidemiológica na avaliação epidemiológica e operacional das atividades de controle da hanseníase. Atuando na coleta, no processamento, na análise e na interpretação dos dados referentes aos casos de hanseníase e seus contatos. Esta realiza ainda, a produção e divulgação das informações que subsidiam análises e avaliações da efetividade das intervenções e embasam o planejamento de novas ações e recomendações a serem implementadas (BRASIL, 2010).

Para que a hanseníase seja eliminada, faz-se necessário, que a vigilância epidemiológica esteja organizada em todos os níveis de atenção, desde nível primário, que se constitui a porta de entrada do sistema de saúde, até o nível ao terciário, de modo a garantir informações sobre a distribuição, a magnitude e a carga de morbidade da doença nas diversas áreas geográficas. Haja vista que, a mesma propícia o acompanhamento rotineiro das principais ações estratégicas para o controle da hanseníase (BRASIL, 2010).

O atual modelo de assistência ao portador de hanseníase deve ser garantido em todos os aspectos, incluindo desde o diagnóstico e tratamento, que é realizado nas unidades básicas de saúde, até as ações de reabilitação física, visando à eliminação do agravo (BRASIL, 2010; FREITAS, 2010).

Destaca-se também, no que tange a assistência ao portador de hanseníase, o papel do enfermeiro como profissional capacitado a prestar um cuidado integral e humanizado a esta população. Cabe a este profissional, atribuições específicas a esta população, como: realizar as visitas domiciliares, exames periódicos de contatos de pacientes hansênicos e implementação da vacinação por BCGid e a capacitação dos agentes comunitários de saúde (ACS) para realização de busca ativa dos casos (BRASIL, 2008).

Sabe-se que a capacidade de julgamento clínico da hanseníase e a tomada de decisão do enfermeiro dependem do seu conhecimento científico, experiência e aprimoramento constante (PEREIRA et al., 2008).

Nesse sentido, faz-se necessário que o enfermeiro esteja ciente da situação epidemiológica do município no qual exerce o seu trabalho, visto que, estes dados servem para subsidiar ações de controle e prevenção da endemia.

Portanto, em virtude da prevalência de hanseníase em Goiás e pelo déficit de estudos 
que abordem essa situação, especificamente no município de Aparecida de Goiânia, este estudo visa contribuir com a monitorização da doença e com o fortalecimento da vigilância epidemiológica ao caracterizar a tendência e a magnitude da doença no município.

Diante do exposto, este estudo tem por objetivo apresentar o perfil epidemiológico da hanseníase no município de Aparecida de Goiânia-GO nos anos de 2006 a 2011.

\section{METODOLOGIA}

Trata-se de um estudo descritivo, baseado em dados do SINAN da Secretaria Municipal de Saúde do Município de Aparecida de Goiânia (SMS), referentes ao período de 2006 a 2011.

A coleta de dados foi realizada de acordo com orientações contidas no Manual de Operação do SINAN. Foi utilizado o método de cálculo preconizado na portaria do MS $n^{\circ} 3.125 / 2010$. Os dados populacionais para o cálculo da taxa anual de detecção foram obtidos a partir do portal do Instituto Brasileiro de Geografia e Estatística (IBGE).

A consolidação dos dados foi realizada com a finalidade de se obter os seguintes índices: coeficiente geral de detecção de casos novos de hanseníase por 100 mil habitantes; coeficiente geral de prevalência por $10 \mathrm{mil}$ habitantes; percentagem de casos curados de hanseníase nos anos estudados e percentual dos casos confirmados de hanseníase por tipo de saída abandono.

Para análise dos dados, utilizaram-se os indicadores epidemiológicos e operacionais do MS e os parâmetros preconizados na portaria 3.125/2010.

Os bancos dos dados disponibilizados pela SMS de Aparecida de Goiânia, utilizados neste estudo, foram filtrados e desvinculados da identificação dos pacientes antes do levantamento das variáveis para garantir o anonimato e a confiabilidade.

\section{RESULTADOS E DISCUSSÃO}

\section{PERFIL EPIDEMIOLOGICO DA} HANSENÍASE EM APARECIDA DE GOIÂNIA- GOIÁS

O Estado de Goiás apresenta uma população estimada de 6.004.045 habitantes distribuídos em 246 municípios, segundo IBGE, censo de 2011. E apresenta coeficiente de detecção de casos novos de hanseníase de 25,55/100 mil habitantes, configurando-se com parâmetros muito altos de acordo com indicadores epidemiológicos do MS (BRASIL, 2011).

Ainda, conforme IBGE (2011), o município de Aparecida de Goiânia possui uma população de 465.093 habitantes. Neste município, entre período de 2006 a 2011, foram detectados 1.404 casos novos de hanseníase, sendo assim, o coeficiente de detecção apresentou variações de 35,6/100 mil habitantes a 60,5/100 mil habitantes, com manutenção da incidência de casos novos 
com parâmetros hiperendêmicos de 2006 a 2010. Já em 2011, os parâmetros configuraram-se como muito alto. Como se pode perceber nos dados apresentados no gráfico1, que mostra a incidência dos casos novos de hanseníase e os coeficientes de detecção anual.
Quanto ao coeficiente de detecção anual de casos novos por 100.000 habitantes, o MS preconiza os seguintes parâmetros: hiperendêmico (_>40,00/100.000 hab.); muito alto (entre 20,00 e 39,99/100.000 hab.); alto (entre 10,00 e 19,99/100.000 hab.); médio (entre 2,00 e 9,99/100.000 hab.) e baixo (< 2,00 /100.000 hab.) (BRASIL, 2010).

Gráfico 1- Detecção Geral de Casos Novos Hanseníase por 100 mil habitantes no Município de Aparecida de GoiâniaGoiás, 2006 a 2011.

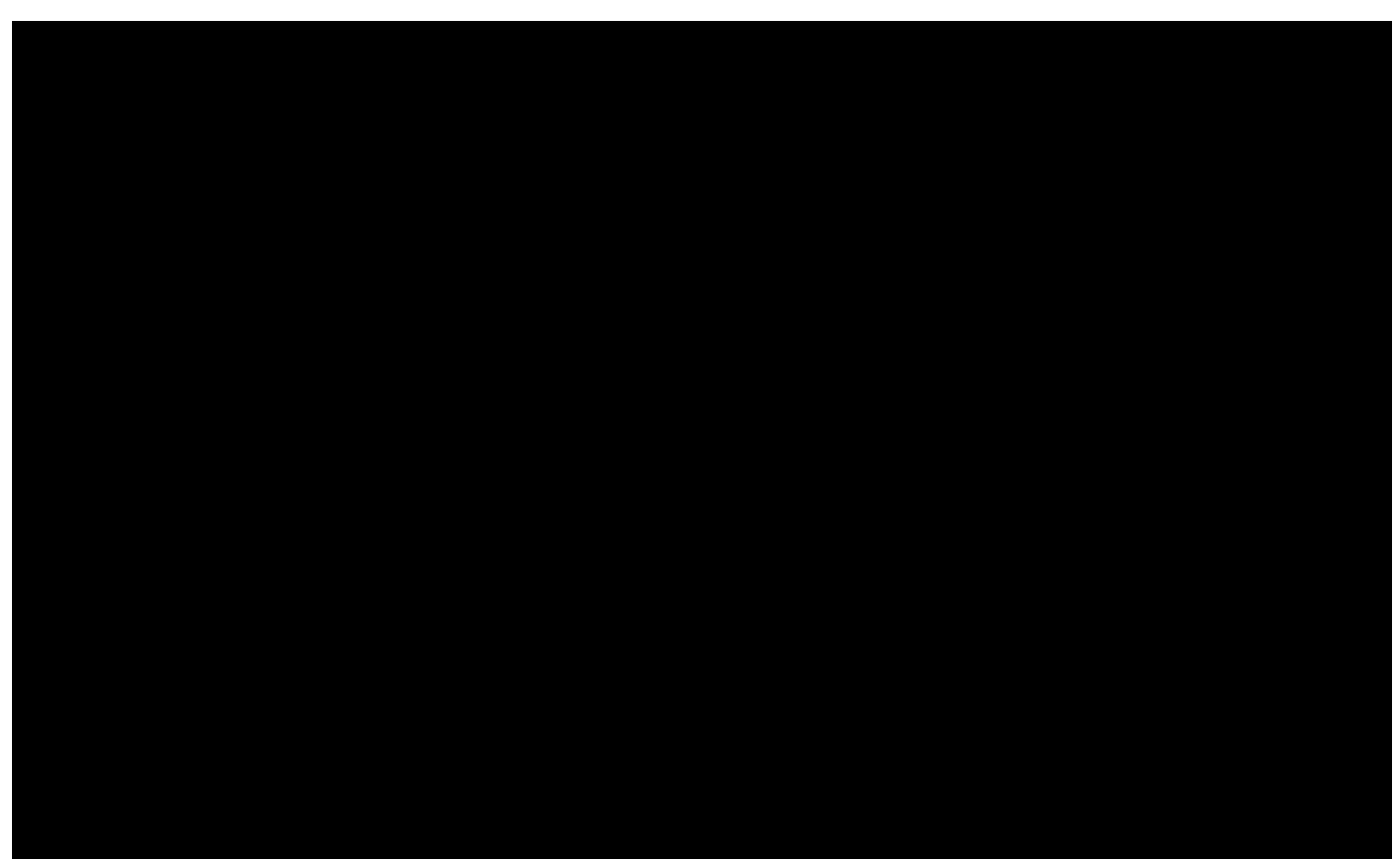

Fonte: SINAN/SVS/MS. Dados preliminares disponível em 14/02/2012, sujeito a alteração.

Observa-se que, entre o período de estudo, de 2006 a 2011, o maior número de infecção por hanseníase no município de Aparecida de Goiânia ocorreu no ano de 2007. Percebe-se que, nos anos subsequentes houve uma diminuição do número de casos, com destaque para o ano de 2011, no qual o coeficiente de detecção apresentou diminuição de $41 \%$, comparado ao ano de 2007.
Nos anos 2006 a 2010 não houve alteração no perfil hiperendêmico no município de Aparecida de Goiânia. Apesar da queda significativa no ano 2011 , este fato mostrou que ainda é preocupante a situação da endemia no município estudado. Haja vista que, no ano de 2011, o município ainda apresenta parâmetro "muito alto" de casos novos da hanseníase. 
Neste contexto, o fato de apresentar queda significativa no número de casos novos de hanseníase no município de Aparecida de Goiânia, pode-se relacionar a maior efetividade de controle desta patologia por meio dos profissionais de saúde e facilidade de acesso do usuário ao atendimento primário.

O gráfico 2, mostra a prevalência da endemia hansênica no município de Aparecida de Goiânia, nos anos de 2006 a 2011. Estes dados são referentes aos casos existentes, residentes em curso de tratamento nos anos estudados, ou seja, o total de casos prevalentes registrados durante o estudo.

O MS apresenta os seguintes parâmetros para o coeficiente de prevalência de casos por 10.000 habitantes: hiperendêmico (_> 20,0 /10.000 hab.); muito alto (entre 19,9 e 10,0/10.000 hab.); alto (entre 9,9 e 5,0/10.000 hab.); médio (entre 1.0 e 4,9 e /10.000 hab.) e Baixo (< 1,0 /10.000 hab.) (BRASIL, 2010).

Gráfico 2- Detecção Geral de Casos Prevalentes de Hanseníase por 10.000 habitantes no Município de Aparecida de Goiânia-Goiás, 2006 a 2011.

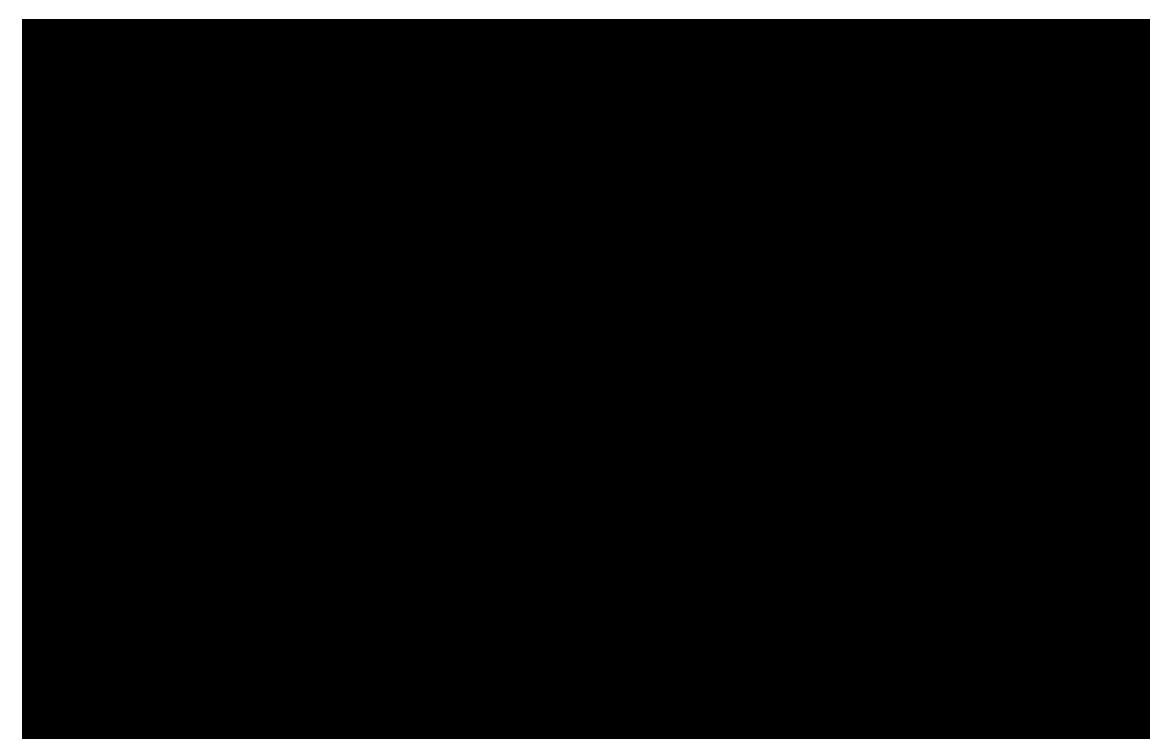

Fonte: SINAN/SVS/MS. Dados preliminares disponível em 14/02/2012, sujeito a alteração.

Observa-se que, entre o período de estudo, de 2006 a 2011, o maior número de prevalência da endemia Hansênica no município de Aparecida de Goiânia ocorreu no ano de 2006. Percebe-se que, nos anos subseqüentes houve uma diminuição da prevalência, com destaque para o ano de 2011, no qual tal coeficiente apresentou praticamente a metade que o coeficiente do ano de 2006.

O perfil epidemiológico da endemia no município de Aparecida de Goiânia, era considerado alto nos anos de 2006 a 2010, evidenciando a manutenção da prevalência de casos nestes anos. Em contrapartida, no ano 
de 2011, houve uma queda significativa, alcançando parâmetros médios.

Apesar da melhora, na apresentação da prevalência de Hanseníase no município de Aparecida de Goiânia, no ano de 2011, este coeficiente apresentado ainda está longe de alcançar a meta do MS que busca a erradicação da hanseníase com o coeficiente de prevalência $<1,0 / 10$ mil habitantes.
O Gráfico 3 apresenta a percentagem de cura entre os casos novos de hanseníase nos anos das COORTES no Município de Aparecida de Goiânia de 2006 a 2011.

Com relação à proporção de cura entre os casos novos de hanseníase os seguintes parâmetros são analisados: Bom (_> 90\%); Regular (entre 75-90\%); Precário (< 75\%) (BRASIL, 2010).

Gráfico 3 - Percentagem de Cura entre os casos novos de Hanseníase nos anos das COORTES no Município de Aparecida de Goiânia - Goiás, 2006 a 2011.

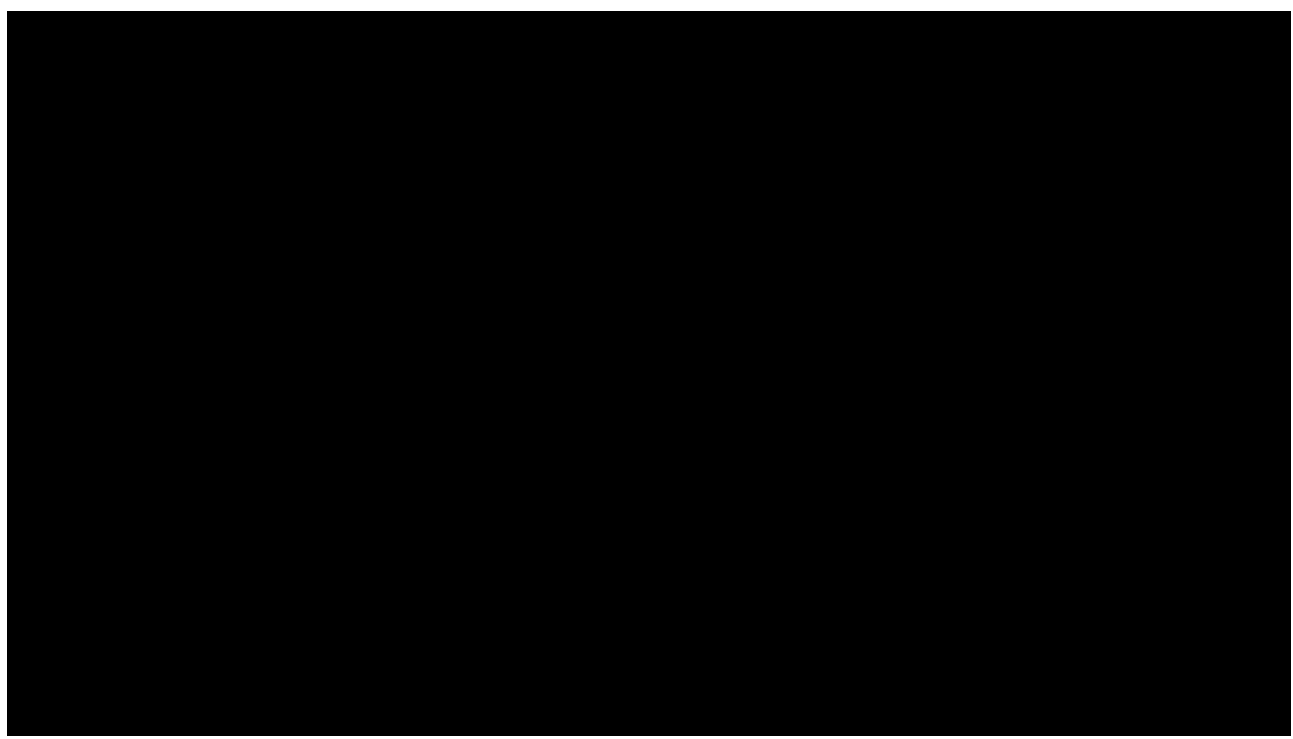

Fonte: SINANNET/SSIS/GVE/SPAIS/SES-APARECIDA, dados sujeitos a alterações

Os percentuais de curas de hanseníase nos anos de 2006, 2007 e 2009 se mantiveram em parâmetros regulares. Já os anos de 2008 e 2010 merecem destaque, visto que, os percentuais de cura tiveram uma queda, apresentando percentual de $74 \%$ e $52 \%$, respectivamente, sendo considerados precários, evidenciando assim, uma queda de cura.

Assim, apesar de investimentos financeiros e científicos empregados na busca da cura da hanseníase e na expectativa de melhoras com o passar dos anos, nota-se que o ano de 2010 não superou a expectativa de cura em relação a esta patologia.

Apesar da queda no percentual de cura no ano de 2010, no ano subsequente, 2011, houve uma grande melhora neste parâmetro, visto que, alcançou o índice de $92 \%$ de casos curados, atingindo o parâmetro considerado bom pelo MS. 
Estes resultados permitem medir a qualidade do atendimento nos serviços de saúde e monitorar os resultados das ações de controle da hanseníase no município estudado.

O gráfico 4 mostra o percentual de casos que abandonaram o tratamento da hanseníase no período estudado.
Conforme Brasil (2010) considera-se abandono quando não foi administrada ao paciente nenhuma dose do tratamento preconizado durante 12 meses consecutivos.

Assim, é fundamental a identificação dos faltosos, realizar a visita domiciliar e busca ativa desses casos, com intuito de evitar que os mesmos venham abandonar o tratamento (BRASIL, 2010).

Gráfico 4 - Percentual dos Casos Confirmados de Hanseníase por tipo de saída abandono no Município de Aparecida de Goiânia - Goiás, 2006 a 2011.

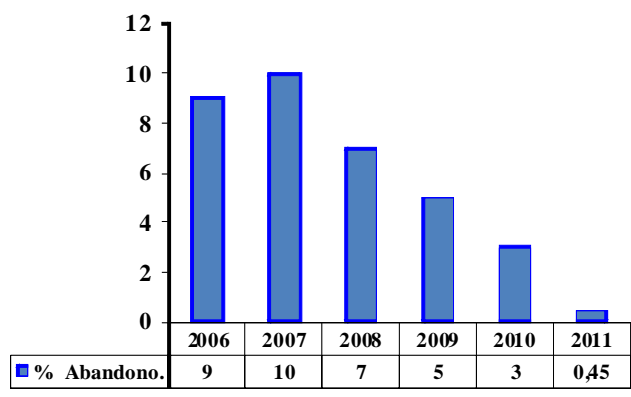

Fonte: SINANNET/SSIS/GVE/SPAIS/SES-APARECIDA, dados sujeitos a alterações

Para análise dos dados de abandono, deve-se considerar, os parâmetros preconizados pelo MS que considera como Precário (_> 25\%); Regular (entre 10 e 24\%); Bom $(<10 \%)$.

O gráfico 4 mostra que no ano de 2006 o percentual foi de $9 \%$, em contrapartida em 2007 apresentou um leve aumento para $10 \%$. Contudo, nos anos subsequentes houve um declínio significativo, chegando em 2011 com o índice de $0,45 \%$, apresentando parâmetros considerados como bom.

O parâmetro considerado bom em todos os anos analisados pode relacionar-se à investimentos para a melhoria da qualidade da assistência e a intensificação de ações de controle da hanseníase.

A Atenção Básica de Saúde, por meio da Estratégia de Saúde da Família (ESF), e PACS, tem atuação imprescindível no controle da evasão ao tratamento da 
hanseníase, visto que, é preconizado pelo MS que, quando o paciente em tratamento de hanseníase começa a faltar nos dias aprazados, deve-se notificar o ACS e o enfermeiro responsável pela equipe, objetivando ações de prevenção de casos de abandono (BRASIL, 2006).

As principais causas de abandono, segundo o MS (2006) são: mudança de área de cobertura do cliente, o cliente que começa a se tratar em outra unidade de saúde, efeitos colaterais desagradáveis e atribuição dos mesmos ao tratamento, o cliente acredita estar totalmente curado, falta de confiança no serviço de saúde ou serviços não disponíveis.

Diante do exposto, o enfermeiro responsável pelo programa de hanseníase, deve ser capacitado para lidar com essas problemáticas, prevenindo possíveis abandonos ao tratamento. Para tanto, este profissional deve acompanhar de maneira integral e humanizada o portador de Hanseníase, prestando-lhe uma assistência de excelência e apresentando-se disponível para sanar qualquer tipo de dúvidas e anseios durante o tratamento.

\section{CONSIDERAÇÕES FINAIS}

O estudo confirmou a hiperendemia da hanseníase no município de Aparecida de Goiânia diante dos resultados de incidência.

Com relação à prevalência da hanseníase no período estudado, observou-se que de 2006 a 2010, houve manutenção da endemia. Entretanto, no ano 2011, houve uma queda significativa de casos prevalentes. Fato que possibilitou a alteração do perfil epidemiológico da endemia no município de Aparecida de Goiânia que era considerado alto nos anos de 2006 a 2010, alcançando parâmetros médios em 2011.

Neste contexto, pode-se constatar que as ações de controle no ano de 2011 foram efetivas. Entretanto, o coeficiente de prevalência no ano referido ainda esta longe de alcançar a meta do MS que busca o coeficiente de prevalência $<1,0 / 10$ mil habitantes, objetivando a erradicação da hanseníase no Brasil.

O estudo mostrou que o percentual de abandono durante o tratamento esta dentro dos limites aceitáveis pelo MS.

Quanto ao percentual por cura, observou-se que no período em estudo houve uma ascensão. Tal fato pode justifica-se, por uma possível melhora na organização da Atenção Básica, com aumento da cobertura dos PACS e ESF. Uma vez que, as principais medidas que podem quebrar o ciclo de transmissão da doença são realizadas pelos profissionais desses programas, mais precisamente o enfermeiro. Uma vez que, a diminuição dos casos, através da cura que contribui para melhoria dos indicadores.

A atuação do enfermeiro, no programa de hanseníase, deve ser implementada, de maneira satisfatória, a fim de realizar ações preventivas contra a manutenção da endemia, 
objetivando não só a eliminação da doença, mas também a busca do diagnóstico precoce.

Destaca-se a relevância dos sistemas de informações da vigilância em saúde, neste caso, do SINAN, visto que, este representa um componente fundamental da vigilância epidemiológica, proporcionando a tomada de decisão, de planejamento das atividades de controle da doença, bem como na execução do projeto terapêutico.

Neste contexto, destaca a importância de um preenchimento das fichas de investigação e notificação do sistema de informação, de forma correta e fidedigna pelo profissional da saúde e que estes dados sejam alimentados no SINAN. Vale ressaltar que

\section{REFERÊNCIAS}

BRASIL. Ministério da Saúde. Casos de hanseníase por municípios brasileiros.

Brasília, 2012. Disponível em:

<http://portal.saude.gov.br/portal/saude/profis sional/visualizar_texto.cfm?idtxt=31205> .

Acesso em 27/02/2012.

BRASIL. Ministério da Saúde. Casos Novos e Prevalentes de Hanseníase por município e tipos de saída por cura e abandono.

Brasília, 2012. Disponível em:

<http://dtr2004.saude.gov.br/sinanweb/tabnet/ tabnet?sinannet/hanseniase/bases/Hansbrnet.d ef>. Acesso em 14/02/2012. todos os profissionais de saúde, bem como a comunidade, tenham acesso a essas informações.

Diante do exposto, ao considerar que os indicadores avaliados neste estudo apontam para uma manutenção da endemia hansênica no município de Aparecida de Goiânia, assim, faz-se necessário o desenvolvimento de estudos científicos que contemple essa temática no estado de Goiás e especificamente, na cidade de Aparecida de Goiânia. Contudo, é fundamental que haja intensificação das atividades de controle da doença, objetivando desenvolver estratégicas educativas com a participação da comunidade e o compromisso efetivo dos gestores.

BRASIL. Ministério da Saúde. Casos Novos e Prevalentes de Hanseníase por município e tipos de saída por cura e abandono.

Brasília, 2012. Disponível em:

<http://dtr2004.saude.gov.br/sinanweb/tabnet/ tabnet?sinannet/hanseniase/bases/Hansbrnet.d ef>. Acesso em 14/02/2012.

BRASIL. Ministério da Saúde. Portaria SVS/SAAS/MS no 3.125, de 07 de outubro de 2010. Brasília, 2010.

BRASIL. Ministério da Saúde. Ações de Controle da Hanseníase na Atenção Básica à Saúde- Manual de Treinamento. Belém-PA, 2006. 
BRASIL. Ministério da Saúde. Casos novos e coeficiente de detecção da hanseníase no

Brasil e estados. Brasília, 2012. Disponível em: 〈http//www.saude.gov.br.svs>. Acesso em: 13 fev. 2012.

BRASIL. Ministério da Saúde. Departamento de Vigilância em Saúde. Caderno de Atenção Básica $n^{\circ} 21,2^{a}$ edição revisada. Brasília- DF. 2008.

BRASIL. Ministério da Saúde. Doenças

Infecciosas e Parasitárias, Brasília, 2008.

BRASIL. Ministério da Saúde. Situação

Epidemiológica Hanseníase Brasil. BrasíliaDF, 2011.

CUNHA, M. D.; SANTOS, R. S.; MATOS, H. J.; OLIVEIRA, M. L. Aspectos epidemiológicos da hanseníase: uma abordagem espacial. Cad. Saúde

Pública, Rio de Janeiro, v. 28, n.6, 2012.

FAÇANHA, M. C.; PINHEIRO, A. C.;

LIMA, J. R. C.; FERREIRA, M. L. L.T.;

TEIXEIRA, G. F. D.; ROUQUAYROL, M.

Z. Investigação Clínica, Epidemiológica,

Laboratorial e Terapêutica. Anais Brasileiros

de Dermatologia. Rio de Janeiro,

v.81, n.4, 2006.

FREITAS, D. Perfil Epidemiológico da Hanseníase no Estado de Goiás. Goiânia, 2010. Disponível em:

<www.sgc.goias.gov.br/upload/links/arq_725 _hanseniase2010.doc>. Acesso em: $15 \mathrm{fev}$ 2012.

IBGE. INSTITUTO BRASILEIRO DE GEOGRAFIA E estatístiCA. Censo de 2000 a 2011.

JUNIOR, A. F. R.; VIEIRA, M. A.;

CALDEIRA, A. P. Perfil Epidemiológico da Hanseníase em uma cidade endêmica no Norte de Minas Gerais. Rev. Sociedade Brasileira de clinica médica, São Paulo, v.10, n.4, 2012.

PEREIRA, S. V. M.; BACHION, M. M.; SOUZA, A. G. C.; VIEIRA, S. M.

S. Avaliação da Hanseníase: relato de experiência de acadêmicos de enfermagem. Rev. Bras. Enferm. Brasília, v.61, n. esp., 2008. 